\title{
Sequence and phylogenetic analysis of S1, S2, M, and N genes of infectious bronchitis virus isolates from Malaysia
}

\begin{abstract}
Two Malaysian infectious bronchitis virus isolates, MH5365/95 and V9/04 were characterized based on sequence and phylogenetic analyses of S1, S2, M, and N genes. Nucleotide sequence alignments revealed many point mutations, short deletions, and insertions in S1 region of both IBV isolates. Phylogenetic analysis of S1 gene and sequences analysis of M gene indicated that MH5365/95 and V9/04 belong to non-Massachusetts strain. However, both isolates share only $77 \%$ identity. Analysis based on S1 gene showed that MH5365/95 shared more than $87 \%$ identity to several Chinese strains. Meanwhile, V9/04 showed only $67-77 \%$ identity to all the previously studied IBV strains included in this study suggesting it is a variant of IBV isolate that is unique to Malaysia. Phylogenetic analysis suggests, although both isolates were isolated 10 years apart from different states in Malaysia, they shared a common origin. Analysis based on S2 and N genes indicated that both strains are highly related to each other, and there are fewer mutations which occurred in the respective genes.
\end{abstract}

Keyword: Infectious bronchitis virus, S1, S2, M and N gene sequences, Phylogenetics analysis 